\title{
Experimenting and Improving Perception of 3D Rotation-Based Transitions between 2D Visualizations
}

\author{
Maxime Cordeil ${ }^{1}$, Christophe Hurter $^{1}$, and Stéphane Conversy ${ }^{2}$ \\ ${ }^{1}$ DSNA/DTI R\&D, ENAC and IRIT/IHCS, \\ ${ }^{2}$ ENAC and IRIT IHCS \\ 7 Avenue Edouard Belin 31055 Toulouse \\ cordeil@cena.fr, \\ chirstophe.hurter@aviation-civile.gouv.fr, \\ stephane.conversy@enac.fr
}

\begin{abstract}
Exploring a multidimensional dataset with visualization requires to transition between points of view. In order to enable users to understand transitions, visualization can employ progressive 3D rotations. However, existing implementations of progressive 3D rotation exhibit some perception problems with visualization of cluttered scene. In this paper, we present a first experiment showing how existing 3D rotation is effective for tracking marks, and that cluttered scenes actually hinder perception of rotation. Then, we propose to set the axis of rotation on the graphical marks of interest, and ran a second experiment showing that focus-centered rotation improves perception of relative arrangement.
\end{abstract}

Keywords: Information Visualization, Visual exploration, Navigation, Transition, 3D Rotation.

\section{Introduction}

Air Traffic Control analysts have to analyze how a particular Air Traffic Controller proceeded to resolve a past conflict involving two aircraft. For these incidents analysis, they use two 2D-views presenting aircraft trajectories from top and vertical views. Thereby, they switch between these views in order to understand the resolution of the conflict, i.e. which aircraft has changed its flight level or heading.

The switch between views can use 3D rotation, a transition that supposedly helps track moving marks and their relative arrangement during the time of the transition. This type of animated transition consists of displaying a temporary 3D view that rotates like a transparent dice. It is used for example for exploration of scatterplots (ScatterDice [1]) or aircraft trajectories (FromDaDy [2]). The scatterdice-like transition implemented in visualization tools used by ATC analysts is slightly different; to explore data, users are free to pan and zoom 2D-views of trajectories. Since the rotation axis is always set at the $\mathrm{X}, \mathrm{Y}, \mathrm{Z}$ center of the data set, the scatterdicelike transition can provoke a loss of focus on these trajectories. Furthermore, in the original usage of scatterdice users deal with a small amount of data, whereas ATC analysts have to deal with a large amount of trajectories (up to 20,000), resulting in 
cluttered scenes. Previous studied benefits of animated transitions showed that rotation can help track objects [3] but the studies did not aim at assessing the perception of relative arrangement.

In this paper, we first present an experiment showing that $3 \mathrm{D}$ rotation can help users tracking objects and that cluttered scenes hinder perception of rotation. Then we propose an improvement of 3D rotation regarding problems we identified, and ran experiments showing that focus-centred rotation improves perception of relative spatial arrangement.

\section{Experiment 1: Tracking Objects}

The goal of this experiment is to validate that animated rotation helps users tracking graphical objects, and that density influences the effectiveness at this task. The underlying hypothesis is: (H1) higher density levels will negatively affect tracking objects.

The screen is a standard 21" LCD screen. A trajectory is depicted with a 1 pixelwidth line. There were nine subjects, all regular computer users (researchers and PhDs in computer science, ergonomics, air traffic controllers), with an age ranging from 22 to 55 (average of age 40). Half of them are familiar with 3D environments. None are Infovis experts. Though the task is related to the specific activity, it does not require Air Traffic Control skills.

In this experiment, the system first presented a top view of green trajectories, with a particular red trajectory (the focus trajectory). After four seconds, the red trajectory turned to green and the system played a 3D-rotation from the top to the vertical view. In this last view, the same trajectory or another one was re-highlighted in red. Then, participants were asked to answer "Yes" or "No" to the question: "Is the red trajectory the same as the red one at the beginning of the animation?" During this first experiment, there were four levels of density (i.e. number of displayed trajectories): Empty (only the two trajectories of interest), Low (10 trajectories), Medium (20 trajectories) and Heavy (40 trajectories). The starting location of the trajectories is at the edge of the screen. We excluded the centre location since we assume that it helps the subject to track a mark. One of our concerns was that subjects would recognize trajectory configurations, and base their answers on previous trials. To avoid this effect, we varied the profiles of the trajectories, and the exact starting location. In summary, we tested 4 levels of density, with 4 trajectory profiles, at 4 locations. Thus, each subject performed 64 trials. The order of conditions was counter-balanced.

We merged the results according to density level and performed a measure-repeated ANOVA on the data. We only consider findings significant if the $\mathrm{P}$ value is less than .05. We first found that the level of density influences the correctness of the users' answers $(\mathrm{p}=, 000)$. In the "empty" condition, users answered $88 \%$ correctly, but only $40 \%$ when the view is cluttered. Wrong answers vary with density accordingly, but the analysis is not significant $(\mathrm{p}=, 055)$. The amount of undecided answers is correlated to density levels $(\mathrm{p}=, 000)$. Users were undecided for only $1 \%$ of the trials with no density, as opposed to $40 \%$ with the cluttered one. 
The experiment shows that H1 was confirmed: the level of density negatively impacts the effectiveness at tracking marks. In the next section, we propose a new rotation design that counters the effect of density.

\section{Experiment 2: Improving 3D Rotation Perception and Perception of Relative Arrangement}

If designers of visualization software want to make rotation effective at tracking marks in dense scenes, they have to provide specific features that counter the density effect. Our proposal is to set the rotation axis on the graphical mark of interest. We called this 3D-rotation a focus-centred rotation.

The experiments we show in this section are not about testing if focus-centred rotation is better than screen-centred rotation at tracking marks. However, perceiving arrangement of marks (i.e. understanding spatial relationship between marks) relies on correct tracking; considering experiment 1 results, we already know that density hinders perceiving arrangement. Thus, these experiments aim to show that a focuscentred 3D rotation helps better the perception of relative arrangement than a screencentred 3D rotation. To summarize, the assumptions we make are: (H1) Focus-centred rotation provides a better accuracy than screen-centred rotation (H2) Density levels have a negative impact on accuracy; (H3) Density levels have less of a negative impact on accuracy with focus-centred rotation compared to screen-centred rotation.

The experimental apparatus and participants are the same as in the first experiment. In this experiment, two trajectories are displayed as lines. When seen in the top view, lines cross. However, one trajectory stays at the same altitude, whereas the other one changes its altitude. The user has to figure out if the stationary trajectory goes under or above the other one. This task requires the user to focus on the trajectories of interest, and to perceive the relative arrangement of the lines. Participants first see a top view with two highlighted crossing trajectories in red (the surrounding trajectories are green). After 4 seconds, the colour of the specific trajectories turns back to green, and the $3 \mathrm{D}$ rotation from the top to the vertical view is played. At the end of the transition, participants were asked to answer the question: "does the stationary trajectory go under the trajectory that changes altitude?" with "yes", "no", or "I don't know" We used three different density levels (empty, medium with 20 trajectories, high with 40 trajectories), 4 profiles of trajectories, 4 trajectory locations (lop-left, top-right, bottom-left, bottom-right), and 2 types of transition (screen-centred, focused-centred rotation). Since we did not repeat measures, users performed 96 trials each.

We first analyzed the global effect of rotation types without taking into account the context. The rotation types have a significant effect on correct answers: $\mathrm{F}(1$, $10)=12,224, p=, 00576$ and on undecided answers: $F(1,10)=7,8955, p=, 01848$. Since the number of bad answers is very low, the analysis does not show a significant effect on the wrong answers: $\mathrm{F}(1,10)=1,5432, \mathrm{p}=, 24248$. This analysis shows that without taking into account the context density, the Focus-centered rotation improves the rotation efficiency to understand the data structure $(\mathrm{H} 1)$.

We also assessed the impact of the context with repeated measures ANOVA using contexts (Empty, Medium, and Heavy) and the type of rotation (Screen-centered, 
Focus-centered) factors. For the participant's correct answers, we have found that there is a main effect of rotation type: $F(2,20)=12,224 \mathrm{p}=, 005762$, and a main effect of context : $F(2,20)=26,395 \mathrm{p}=, 000$. We also found an interaction between density and rotation type $(\mathrm{F}(2,20)=6,754 \mathrm{p}=, 005740)$. These results show that density has less of an impact on correctness of answers with focus-centered rotation (from $99 \%$ to $90 \%$ vs from $99 \%$ to $66 \%$ for screen-centered rotation).

For wrong answers, we have found that there is no main effect. Results were not found significant for the rotation type effect $(\mathrm{F}(2,20)=1,53918 \mathrm{p}=, 243058)$ and the density effect $(\mathrm{F}(2,20)=1,51103 \mathrm{p}=, 244827)$. We did not found an interaction between density and rotation type $(\mathrm{F}(2,20)=0,61053 \mathrm{p}=, 0552848)$. These results are not significant because participants gave very few wrong answers. This suggests that both transitions are not misleading. For the participants' undecided answers, we have found that there is a main effect of rotation type $(F(2,20)=7,89552 \mathrm{p}=, 018478)$ and a main effect of context $(\mathrm{F}(2,20)=13,60981 \mathrm{p}=, 000186)$. We also found an interaction between density and rotation type $(\mathrm{F}(2,20)=9,25000 \mathrm{p}=, 001431)$.

We can conclude that Focus-centered rotation minimizes the indecision of participants when density increases (for heavy density, $8 \%$ vs $28 \%$ undecided answers). Our analysis shows that the type of rotation has a significant effect on the user's ability to understand the data structure $(\mathrm{H} 2)$ and that the impact of the context density is reduced with the Focus-centered rotation (H3). Hence the Focus-centered rotation is more efficient than the Screen-centered rotation.

\section{Conclusion and Design Perspective}

We proposed that rotation-based transition help in tracking marks, and perceiving their relative arrangement. We showed that users can track marks with a rotation, but that density hinders it. We also showed that rotation enables users to perceive relative arrangement, and that focus-centred rotation improves perception of relative arrangement compared to a screen-centred rotation. As stated before, this paper is about perception of rotation for visualization, and not about the interaction with the rotation. Now that we know that perception is better with a focus-centred rotation, we need to enable the user to rotate around this axis instead of the centre of the screen. Of course, in contrast to experiment 2, the system does not know which marks the user currently analyses. Hence, we are currently working on the design of an interaction to set the location of the axis. Solutions based on selecting an object are not suitable, since our scenes are very dense and cluttered, which makes pointing a difficult task.

\section{References}

1. Elmqvist, N., Dragicevic, P., Fekete, J.-D.: Rolling the dice: Multidimensional Visual Exploration using Scatterplot Matrix Navigation Visualization. IEEE Trans. On Visualization and Computer Graphics 14(6), 1148-1539 (2008)

2. Hurter, C., Conversy, C., Tissoires, B.: FromDaDy: Spreading Aircraft Trajectories Across Views to Support Iterative Queries. IEEE Transactions on Visualization and Computer Graphics 15(6), 1017-1024 (2009)

3. Robertson, G., Cameron, K., Czerwinski, M., Robbins, D.: Animated visualization of multiple intersecting hierarchies. Information Visualization 1(1), 50-65 (2002) 